\title{
Anti-Xa Inhibitor-Induced Hemorrhagic Pruritic Rash: A Case Report on Possible Cross-Reactivity Between Apixaban and Rivaroxaban
}

\section{Takla R Anis \\ Whitney Jandreau}

Pharmacy Department, Northern Light Eastern Maine Medical Center, Bangor, ME, USA
Correspondence: Takla R Anis Pharmacy Department, Northern Light Eastern Maine Medical Center, 489 State Street, Bangor, ME, 0440I, USA

Email takla.anis@gmail.com

\begin{abstract}
Direct oral anticoagulants (DOACs) have gained increasing popularity in clinical practice in the past decade. While DOACs have fewer associated adverse events as compared to warfarin, DOAC-induced hypersensitivity is a rare adverse event that has been reported in the literature. We describe a case of apixaban-induced hemorrhagic pruritic rash in a 62-yearold man with protein $\mathrm{C}$ deficiency for which he was receiving warfarin. During hospitalization for another issue, his anticoagulant was converted to apixaban. Within 6 hours of receiving the first dose of apixaban, he developed a hemorrhagic pruritic rash around his buttocks area and extending into his groin. The following day, apixaban was replaced with rivaroxaban, while the rash continued to worsen and spread further around his back. After 3 days of DOAC therapy, the patient was reverted back to warfarin and within 24 hours the rash subsided. This case report indicates a potential rare adverse drug reaction that may become more prominent given the increasing utilization of DOACs in clinical practice. Future research is needed to further investigate this adverse event and ensure that providers and patients are aware of it. Additionally, this report documents to our knowledge, the first report of an immediate hypersensitivity reaction to DOACs.
\end{abstract}

Keywords: apixaban, rivaroxaban, rash, hypersensitivity

\section{Introduction}

Warfarin is a vitamin $\mathrm{K}$ antagonist (VKA) that is commonly used for multiple thromboembolic conditions requiring anticoagulation. A newer class of medications, direct oral anticoagulants (DOACs), is increasingly being recommended over VKA due to fewer bleeding-related complications. Additionally, DOACs do not require frequent lab monitoring and have fewer drug-drug interactions, making them an appealing therapeutic alternative to VKA. Apixaban and rivaroxaban are DOACs indicated for treatment and prevention of venous thromboembolism and stroke risk reduction in patients with nonvalvular atrial fibrillation. ${ }^{1,2}$

With increased use of DOACs over the past decade, there have been increasing reports of adverse reactions, including hypersensitivity reactions, associated with their use. Drug-induced hypersensitivity reactions to apixaban, including skin rash, were reported in the package insert in $<1 \%$ of the patients in approval trials. $^{2}$ Additionally, $2.1 \%$ of the patients who received rivaroxaban during approval trials have experienced pruritus. ${ }^{1}$ In their literature review, Carli et al were able to identify 29 case reports of patients with possible hypersensitivity reactions to DOACs. ${ }^{3}$ This report aims to further increase awareness of the 
potential for DOAC-induced hypersensitivity reactions and encourage pharmacovigilance with this medication class.

\section{Case Description}

Our patient was a 62-year-old white man with poorly controlled diabetes who was admitted to the hospital with a right great toe osteomyelitis and associated Staphylococcus aureus bacteremia secondary to diabetic foot infection. Seven months prior to admission, our patient received a 5-week course of intravenous (IV) cefepime and oral metronidazole for osteomyelitis of the foot. His past medical history was also significant for peripheral vascular disease and protein $\mathrm{C}$ deficiency for which he was on chronic anticoagulation with warfarin. Upon admission, warfarin was held for elevated INR of 4.8. During this hospitalization, he received ceftriaxone which was converted to cefazolin upon receipt of blood culture results. Additionally, he was on subcutaneous enoxaparin, insulin, and oral metronidazole.

On day 6, the patient underwent amputation of his right great toe and continued IV antibiotics with cefazolin 2 g IV three times daily. On day 9, anticoagulation was resumed with apixaban $5 \mathrm{mg}$ twice daily due to a pattern of poor time in therapeutic range with warfarin. Within 6 hours from receiving the first dose of apixaban, the patient developed a violaceous maculopapular rash, vasculitic in appearance, around his upper thighs. Apixaban was switched to rivaroxaban $10 \mathrm{mg}$ by mouth daily. The patient received two doses of rivaroxaban on days 10 and 11, but the rash continued to spread to his buttocks and back area, at which point rivaroxaban was switched to warfarin with an enoxaparin bridge. The following day, the rash began to subside, and the patient was discharged to complete a 6-week course of IV cefazolin. During his 1-week follow-up at the outpatient clinic, there was no evidence of the rash and no further reactions were reported during his subsequent weekly follow-ups.

\section{Discussion}

This report describes a patient on VKA for protein $\mathrm{C}$ deficiency, who, upon transition to apixaban, experienced an immediate hypersensitivity reaction with potential cross-reactivity to rivaroxaban, resolving with resumption of previously tolerated warfarin.

Historically, VKA therapy has been the standard of care for anticoagulation in inherited thrombophilias such as protein $\mathrm{C}$ deficiency. Guidelines from the International
Society of Thrombosis and Haemostasis (ISTH), the American College of Chest Physicians (CHEST), and the American Society of Hematology (ASH) do not make definitive recommendations about anticoagulant selection in thrombophilias. ${ }^{4-6}$ Available literature on the appropriateness of DOACs in inherited thrombophilias is conflicting. Small case series have demonstrated both safety and efficacy of various DOACs in the inherited thrombophilias ${ }^{7-9}$; however, a 2021 literature review concluded there remains insufficient evidence to recommend DOACs in the high-risk inherited thrombophilias, protein $\mathrm{C}$ and $\mathrm{S}$ deficiencies and antiphospholipid syndrome. ${ }^{10}$

As we await a guideline-defined role of DOACs in these patients (of note, the ASH website indicates a forthcoming guideline titled Thrombophilias), for some, it may be preferred to accept the risk of DOAC therapy relative to the challenges associated with VKA therapy described above. For these reasons, providers and patients with thrombophilic conditions may decide together to transition VKA therapy to a DOAC, as was the case with our patient.

In evaluating potential medication causes of this patient's skin rash, beta-lactams and apixaban were considered. The rash was not typical of a beta-lactam rash and the patient had tolerated beta-lactams in the past, including a recent 5-week course of cefepime, and was on betalactam therapy for 8 days prior to receiving apixaban. Apixaban, the other potential culprit, has rarely been associated with skin reactions. Given the temporal relationship of the initiation of apixaban with onset of the rash, and that the patient had tolerated beta-lactams in the past, the rash was attributed to apixaban; as shown in Table 1, a Naranjo score of 7 was calculated, indicating probable causality. ${ }^{11}$ Apixaban-induced rash was diagnosed without biopsy or patch testing as these are not readily available for inpatients at our institution.

A previous case report has shown that a patient who has developed apixaban-induced type I hypersensitivity reaction was able to tolerate rivaroxaban. ${ }^{12}$ Additionally, Sasson et al reported that a patient with rivaroxabaninduced rash was successfully switched to apixaban, which indicates a low potential for cross-reactivity between rivaroxaban and other DOACs. ${ }^{13}$ Thus, a recommendation was made to trial this patient on rivaroxaban in an attempt to avoid warfarin due to a history of labile INRs. The patient received his first rivaroxaban dose about 24 hours from the first apixaban dose. His rash continued to worsen after receiving two doses of rivaroxaban on days 10 and 11 . Possible cross-reactivity between 
Table I Naranjo Adverse Drug Reaction Probability Scale for Apixaban-Induced Rash.

\begin{tabular}{|c|c|c|c|}
\hline Assessment Questions & Yes & No & Do Not Know \\
\hline I. Are there previous conclusive reports on this reaction? & +1 & 0 & 0 \\
\hline 2. Did the adverse drug reaction appear after the suspected drug was administered? & +2 & -1 & 0 \\
\hline 3. Did the adverse drug reaction improve when the drug was discontinued? & +1 & 0 & 0 \\
\hline 4. Did the adverse drug reaction reappear when the drug was readministered? & +1 & 0 & $\mathbf{0}$ \\
\hline 5. Are there alternative causes for this adverse drug reaction? & -1 & +2 & 0 \\
\hline 6. Did the reaction reappear when a placebo was given? & -1 & +1 & 0 \\
\hline 7. Was the drug detected in blood or other fluids in concentrations known to be toxic? & +1 & 0 & $\mathbf{0}$ \\
\hline 8. Was the reaction more severe when the dose was increased or less severe when the dose was decreased? & +1 & 0 & $\mathbf{0}$ \\
\hline 9. Did the patient have a similar reaction to the same or similar drugs in any previous exposure? & +1 & 0 & 0 \\
\hline 10. Was the adverse drug reaction confirmed by any objective evidence? & +1 & 0 & 0 \\
\hline Total score & \multicolumn{3}{|c|}{$7=$ Probable } \\
\hline
\end{tabular}

Notes: Bolded text $=$ score assigned to each question. Reproduced from Naranjo CA, Busto U, Sellers EM, et al. A method for estimating the probability of adverse drug reactions. Clin Pharmacol Ther. 1981;30(2):239-245. @ 198I American Society for Clinical Pharmacology and Therapeutics. "

factor Xa inhibitors has been reported previously in a patient that was challenged with apixaban 7 days after developing delayed hypersensitivity reaction to edoxaban. ${ }^{14}$ Given apixaban's half-life of about 12 hours, we could not definitively confirm that our patient developed cross-reactivity between factor $\mathrm{Xa}$ inhibitors since apixaban would not have been fully cleared when rivaroxaban was initiated. To our knowledge, this is the first case report of hypersensitivity to apixaban occurring within a few hours of receiving the first dose, suggesting that DOAC-induced hypersensitivity reactions are not always delayed. The exact mechanism of this reaction remains unknown. Also, it is unclear whether certain conditions like protein $\mathrm{C}$ deficiency could increase the likelihood of developing this adverse reaction. This reaction prompts the need to monitor patients for rare adverse reactions like skin rash when initiating apixaban and rivaroxaban, and potentially other DOACs like edoxaban and dabigatran. With the increased use of DOACs as first-line treatment for many thromboembolic conditions, this reaction may become more prominent.

\section{Conclusion}

Our primary aim from this report is to help in increasing vigilance and clinician awareness. Future studies will need to be conducted to determine the exact mechanism behind this adverse reaction and whether there are factors that predispose patients to it.

\section{Consent}

Northern Light Eastern Maine Medical Center's institutional review board (IRB) requirement for publication of a case report is to obtain informed consent from the patient; this was completed prior to authoring this report.

\section{Acknowledgments}

We would like to acknowledge the Family Medicine and Infectious Diseases teams at Northern Light Eastern Maine Medical Center for their assistance. We would like to thank Dr Rebekah Gass, MD, Lead Physician of Infectious Diseases, for her review of this report.

\section{Disclosure}

The authors report no conflicts of interest in this work.

\section{References}

1. XARELTO ${ }^{\circledR}$ (Rivaroxaban) Tablets [Package Insert]. Titusville, NJ: Janssen Pharmaceuticals, Inc; 2017.

2. Eliquis [Package Insert]. Princeton, NJ: Bristol-Myers Squibb; December, 2012.

3. Carli G, Farsi A, Chiarini F, Lippolis D, Cortellini G. Hypersensitivity reactions to non-vitamin $\mathrm{K}$ oral anticoagulants - a review of literature and diagnostic work-up proposal. Eur Ann Allergy Clin Immunol. 2019;51(1):7-14. doi:10.23822/EurAnnACI.1764-1489.80

4. Wang T, Zwicker JI, Ay C, et al. The use of direct oral anticoagulants for primary thromboprophylaxis in ambulatory cancer patients: guidance from the SSC of the ISTH. J Thromb Haemost. 2019;17(10): 1772-1778. doi:10.1111/jth.14564

5. Kearon C, Akl EA, Ornelas J, et al. Antithrombotic therapy for VTE disease: CHEST guideline and expert panel report. Chest. 2016;149 (2):315-352. doi:10.1016/j.chest.2015.11.026.

6. Ortel TL, Neumann I, Ageno W, et al. American Society of Hematology 2020 guidelines for management of venous thromboembolism: treatment of deep vein thrombosis and pulmonary embolism. Blood Adv. 2020;4(19):4693-4738. doi:10.1182/bloodadvances.20200 01830

7. Elsebaie MAT, van Es N, Langston A, Büller HR, Gaddh M. Direct oral anticoagulants in patients with venous thromboembolism and thrombophilia: a systematic review and meta-analysis. J Thromb Haemost. 2019;17:645-656. doi:10.1111/jth.14398 
8. Serrao A, Lucani B, Mansour D, et al. Direct oral anticoagulants in patients affected by major congenital thrombophilia. Mediterr J Hematol Infect Dis. 2019;11(1):e2019044. PMID: 31308920; PMCID: PMC6613626. doi:10.4084/MJHID.2019.044.

9. Undas A, Goralczyk T. Non-vitamin K antagonist oral anticoagulants in patients with severe inherited thrombophilia: a series of 33 patients. Blood Coagul Fibrinolysis. 2017;28(6):438-442. PMID: 28079536. doi:10.1097/MBC.0000000000000613

10. Valanejad SM, Davis KA. Direct oral anticoagulants in select patients with hypercoagulable disorders. Ann Pharmacother. 2021;55 (7):891-901. PMID: 33100017. doi:10.1177/1060028020968551.

11. Naranjo CA, Busto U, Sellers EM, et al. A method for estimating the probability of adverse drug reactions. Clin Pharmacol Ther. 1981;30 (2):239-245. doi:10.1038/clpt.1981.154
12. Josyln JA, Khattak FH, Geraci SA. A case of a reversible neurologic adverse reaction to apixaban confirmed by re-challenge. J Clin Med Res. 2018;10(6):523-526. doi:10.147 40/jocmr3394w

13. Sasson E, James M, Russell M, Todorov D, Cohen H. Probable rivaroxaban-induced full body rash: a case report. J Pharm Pract. 2018;31(5):503-506.

14. Cortellini G, Rossi F, Lippolis D, Cortellini F, Gavioli B, Ballardini G. Delayed hypersensitivity to new oral anticoagulants. Demonstration of cross reactivity for the drug category and definition of non-irritant concentrations for patch tests. Eur Ann Allergy Clin Immunol. 2018. doi:10.23822/EurAnnACI.17641489.71

\section{Publish your work in this journal}

Clinical Pharmacology: Advances and Applications is an international, peer-reviewed, open access journal publishing original research, reports, reviews and commentaries on all areas of drug experience in humans. The manuscript management system is completely online and includes a very quick and fair peer-review system, which is all easy to use. Visit http://www.dovepress.com/testimonials.php to read real quotes from published authors. 